\title{
Simplified Input Voltage Sensorless Vector Control for PWM Rectifiers
}

\author{
Kapil Upamanyu, Chitrank Ameta, G Narayanan \\ Department of Electrical Engineering, Indian Institute of Science, Bangalore, India \\ kapilu@iisc.ac.in, chitranka@iisc.ac.in, gnar@iisc.ac.in
}

\begin{abstract}
Input voltage information is essential for vector control of three-phase active pulse-width modulated (PWM) rectifier. Input voltage sensors impact cost, size and reliability of the PWM rectifier. Existing input voltage sensorless methods either involve extensive mathematical computations to estimate the input voltage or have a control structure significantly different from that of conventional vector control. This work proposes an input voltage sensorless control of PWM rectifier which retains the simplicity of vector control and also reduces the computation requirement. Modelling and analysis of the rectifier system with the proposed phase locked loop (PLL) are presented. The design procedure for the PLL is detailed, and is validated through frequency domain studies. Performance of the proposed method is compared with that of conventional sensor based vector control through simulations and experiments. The responses of both are found to be almost indistinguishable while the proposed method is shown to require reduced computational effort.

Index Terms-AC-DC conversion, phase locked loop, PWM rectifier, sensorless control, vector control
\end{abstract}

\section{INTRODUCTION}

Pulse width modulated (PWM) rectifier is one of the most extensively used power electronic converters. Unity power factor AC-DC conversion, grid connected photovoltaic system, wind turbine based energy system and uninterrupted power supply are some of the applications where PWM rectifiers are actively used [1].

A three-phase PWM rectifier (see Fig. 1) typically requires five sensors for its operation and control, namely, two ac voltage sensors, two ac current sensors and one dc voltage sensor. The two ac sensors and the dc sensor are also required for protection apart from control of rectifier. But the input ac voltage sensors can be avoided if the control strategy of the rectifier allows sensorless operation. Elimination of the input voltage sensors results in a number of advantages such as reduced hardware component count, reduced development time, increased robustness, reduced cost, reduced sampling requirements and other feedback related problems arising from distortion and offset present in the sensors [2].

When grid is the ac voltage source to the rectifier, line inductance $L$ is connected between the grid and inverter to reduce the input current ripples. But, when the source is a synchronous generator, the internal inductance is large enough to filter the current ripple. In this case, the equivalent of

This work was supported by the Department of Science and Technology, Government of India, under a Fund for Improvement of Science \& Technology Infrastructure (FIST) project titled "Smart Energy Systems Infrastructure: Hybrid Test Bed".

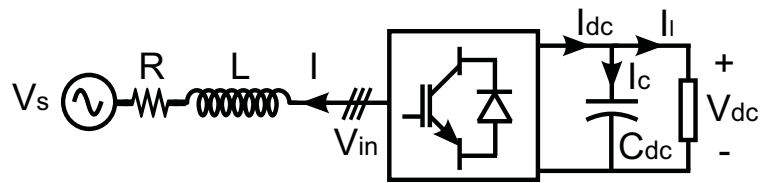

Fig. 1: Circuit diagram of a PWM Rectifier connected to an ac source

grid voltage (required for synchronizing) is the generator internal voltage, which is not available for measurement. Hence sensorless operation is mandatory [3].

Source voltage estimation in [4], [5] involves calculating inductive filter drop through differentiation which is known for noise-related problems in its implementation. To circumvent the problem due to differentiation, certain voltage terms are integrated to estimate the virtual flux [6]-[9], which is then used for sensorless control. Integrators also result in dc-drift and saturation problems, requiring low-pass filters (LPF). The phase-delay and attenuation of the signals caused by these LPFs need suitable compensation [6]. Also methods such as adaptive neural estimator [10] and model predictive based control [11], [12] introduce additional complexity to the design of the control.

The existing input voltage sensorless methods require higher computation effort, more complex control structure and/or additional controllers to be designed/tuned than the conventional sensor-based methods [2]-[12]. This paper proposes a simplified sensorless control method which has a computational requirement even lower than that of the conventional sensor-based method, while providing a comparable dynamic performance.

\section{Vector CONTROL OF PWM RECTIFIER}

Fig. 2 shows a block diagram of vector controlled PWM rectifier in $d q$-domain where input voltage vector is aligned to q-axis [13]. Reactive power is controlled by independent control of d-axis current $I_{d}$. Active power control is achieved by controlling q-axis current $I_{q}$ whose reference $I_{q}^{*}$ is provided by the dc voltage $V_{d c}$ controller. While the inner current control loops have high bandwidth, the outer voltage control loop to regulate $V_{d c}$ has a lower bandwidth.

Transformation of $a b c$ quantities into $d q$ reference frame for vector control requires input voltage vector angle $\theta$. It 


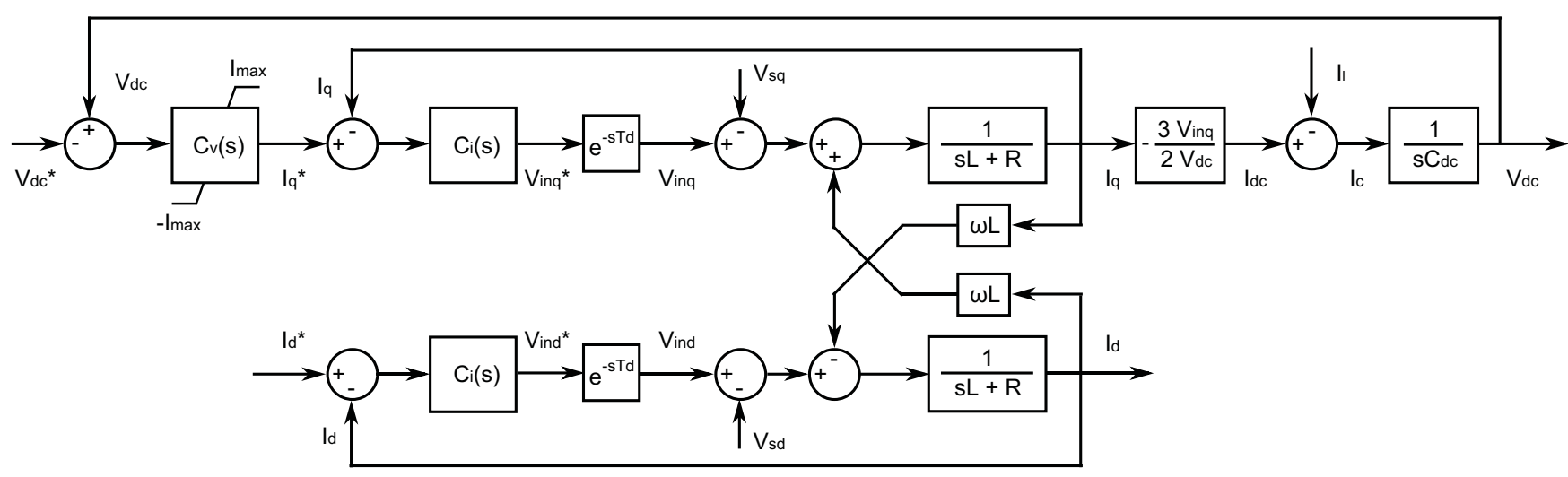

Fig. 2: Block diagram of a vector controlled PWM Rectifier

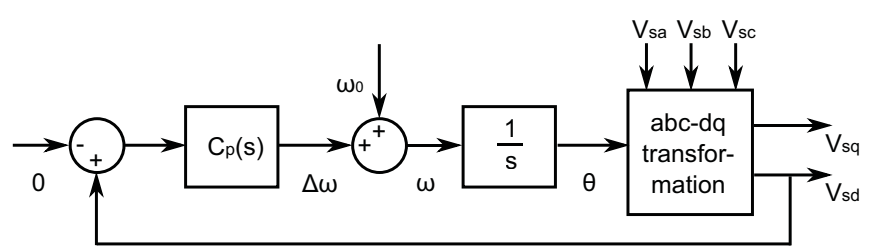

Fig. 3: Block diagram of a conventional synchronous reference frame Phase Locked Loop (PLL)

is obtained using a phase-locked loop (PLL) whose block diagram is shown in Fig. 3.

Design of current controller $C_{i}(s)$, voltage controller $C_{v}(s)$ and PLL controller $C_{p}(s)$ is discussed in section III.

\section{Controller Design for Vector Control}

Controllers are designed for the system whose parameters are given in Table I. Series resistance of filter inductance $R$ is neglected in the design. Delay $T_{d}$ is considered to be twice the switching cycle (i.e. $1 / 2 f_{s w}$ ). Each of $C_{i}(s), C_{v}(s)$ and $C_{p}(s)$ is implemented using proportional-integral (PI) controller whose general transfer function is given by

$$
C_{x}(s)=K_{x} \frac{s+\omega_{x}}{s}, \quad x=i, v, p
$$

\section{A. Current Controller Design}

From the block diagram shown in Fig. 2, q-axis and d-axis currents can be represented as follows:

$$
\begin{aligned}
& I_{q}(s)=T_{i 1}(s) I_{q}^{*}(s)+T_{i 2}(s)\left(V_{s q}(s)-\omega L I_{d}(s)\right) \\
& I_{d}(s)=T_{i 1}(s) I_{d}^{*}(s)+T_{i 2}(s)\left(V_{s d}(s)+\omega L I_{q}(s)\right) \\
& T_{i 1}(s)=\frac{\frac{C_{i}(s) e^{-s T_{d}}}{s L+R}}{1+\frac{C_{i}(s) e^{-s T_{d}}}{s L+R}}, T_{i 2}(s)=-\frac{\frac{1}{s L+R}}{1+\frac{C_{i}(s) e^{-s T_{d}}}{s L+R}}
\end{aligned}
$$

Controller $C_{i}(s)$ should be designed such that the desired transfer function $T_{i 1}$ has close to unity gain and zero phase lag over the frequency range of interest. The disturbance transfer function $T_{i 2}$ should have a gain close to zero.
TABLE I: Hardware and control parameters

\begin{tabular}{|l|l||l|l|}
\hline Parameter & Value & Parameter & Value \\
\hline \hline$L$ & $3 \mathrm{mH}$ & $C_{d c}$ & $1230 \mu \mathrm{F}$ \\
\hline$V_{s}$ & $415 V_{r m s}$ & $\omega$ & $100 \pi \mathrm{rad} / \mathrm{s}$ \\
\hline$K_{p}$ & 1 & $\omega_{p}$ & $10 \mathrm{rad} / \mathrm{s}$ \\
\hline$K_{v}$ & 0.5 & $\omega_{v}$ & $100 \mathrm{rad} / \mathrm{s}$ \\
\hline$K_{i}$ & 10 & $\omega_{i}$ & $1000 \mathrm{rad} / \mathrm{s}$ \\
\hline$I_{\max }$ & $5 \mathrm{~A}$ & $f_{s w}$ & $10 \mathrm{kHz}$ \\
\hline
\end{tabular}

Initial approximations of gain $K_{i}$ and zero frequency $\omega_{i}$ for $C_{i}(s)$ can be obtained as:

$$
K_{i}^{\prime}=\omega_{i b} L, \quad \omega_{i}^{\prime}=\omega_{i b} / 3
$$

where $\omega_{i b}$ is desired current controller bandwidth. Value of $\omega_{i b}$ is generally kept 3-10 times lower than the switching frequency. Values of $K_{i}$ and $\omega_{i}$ can be varied subsequently about their approximated values to fine tune the performance. Too high a value of $K_{i}$ makes the controller unstable whereas selecting a lower value reduces the controller bandwidth. Similarly, lower value of $\omega_{i}$ reduces the frequency range in
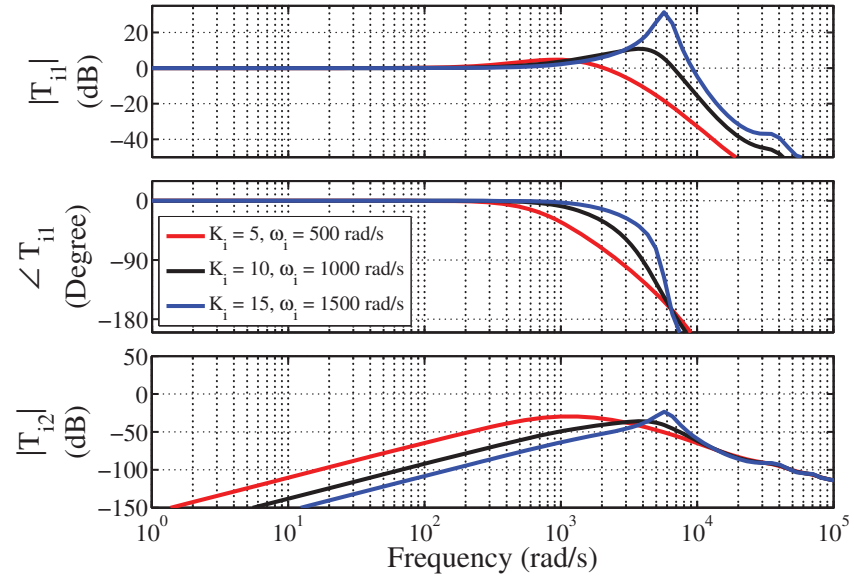

Fig. 4: Bode plots of $T_{i 1}(s)$ and $T_{i 2}(s)$ for current controller design 

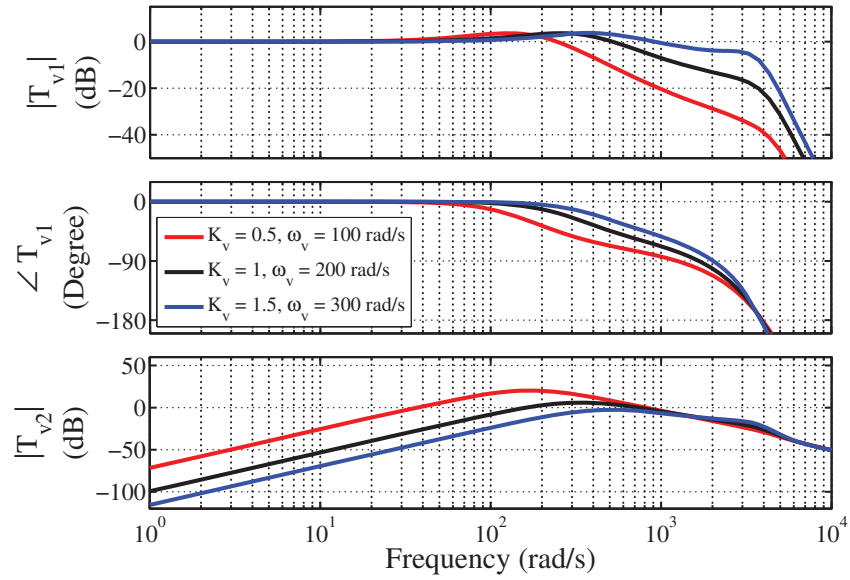

Fig. 5: Bode plots of $T_{v 1}(s)$ and $T_{v 2}(s)$ for voltage controller design

which controller rejects the disturbance, while selecting too high a value makes the system oscillatory.

Frequency responses of $\left|T_{i 1}(j \omega)\right|, \angle T_{i 1}(j \omega)$ and $\left|T_{i 2}(j \omega)\right|$ for three values of $\omega_{i b}$ (i.e. $1500 \mathrm{rad} / \mathrm{s}, 3000 \mathrm{rad} / \mathrm{s}$ and 4500 $\mathrm{rad} / \mathrm{s}$ ) are shown in Fig. 4. $K_{i}=10$ and $\omega_{i}=1000 \mathrm{rad} / \mathrm{s}$ are chosen as the controller parameters as these provide good current reference tracking and disturbance rejection upto 3000 $\mathrm{rad} / \mathrm{s}$.

\section{B. Voltage Controller Design}

The dc bus voltage can be represented as:

$$
\begin{gathered}
V_{d c}(s)=T_{v 1}(s) V_{d c}^{*}(s)-T_{v 2}(s) I_{l}(s) \\
T_{v 1}(s)=\frac{\frac{m C_{v}(s) T_{i 1}(s)}{s C_{d c}}}{1+\frac{m C_{v}(s) T_{i 1}(s)}{s C_{d c}}}, T_{v 2}(s)=\frac{\frac{1}{s C_{d c}}}{1+\frac{m C_{v}(s) T_{i 1}(s)}{s C_{d c}}}, \\
m=\frac{3 V_{s q}}{2 V_{d c}}
\end{gathered}
$$

Controller $C_{v}(s)$ should be designed similar to $C_{i}(s)$. Initial estimates of controller parameters are provided in terms of desired voltage controller bandwidth $\omega_{v b}$ :

$$
K_{v}^{\prime}=\omega_{v b} C / m, \quad \omega_{v}^{\prime}=\omega_{v b} / 3
$$

Value of $\omega_{i b}$ is between $0.1 \omega_{i b}$ and $0.3 \omega_{i b}$. Fig. 5 shows the frequency responses of $\left|T_{v 1}(j \omega)\right|, \angle T_{v 1}(j \omega)$ and $\left|T_{v 2}(j \omega)\right|$ for three values of $\omega_{v b}$ (i.e. $300 \mathrm{rad} / \mathrm{s}, 600 \mathrm{rad} / \mathrm{s}$ and $900 \mathrm{rad} / \mathrm{s}$ ). Controller parameters that are selected are $K_{v}=0.5$ and $\omega_{v}=$ $100 \mathrm{rad} / \mathrm{s}$.

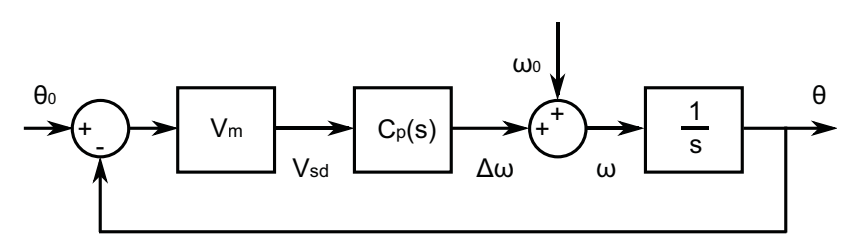

Fig. 6: Linearised model of existing PLL

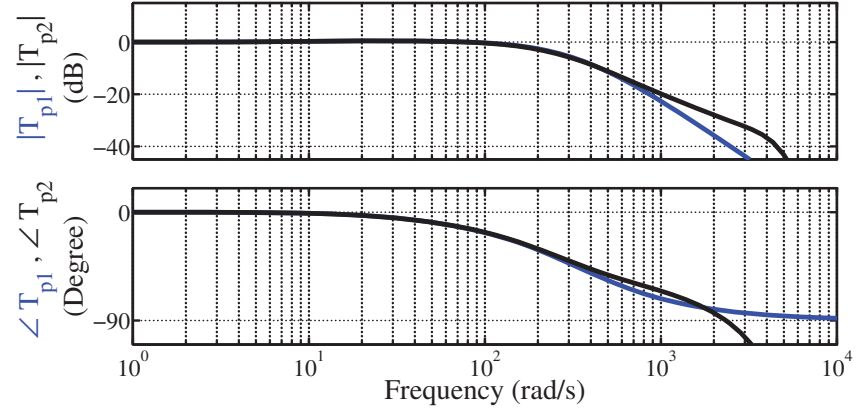

Fig. 7: Bode plots of $T_{p 1}(s)$ and $T_{p 2}(s)$ comparing frequency responses of sensor based PLL and sensorless PLL

\section{PLL Controller Design}

PLL controller loop, shown in Fig. 3, is non-linear due to presence of $a b c-d q$ transformation block [13]. The block needs to be linearised about the operating point. Considering $V_{m}$ to be the magnitude and $\theta_{0}$ to be the phase of input voltage vector, $a b c-d q$ transformation block provides orthogonal projections of the input voltage vector after rotating it by angle $\theta$ in opposite direction. Hence,

$$
v_{s q}=V_{m} \cos \left(\theta_{0}-\theta\right), \quad v_{s d}=V_{m} \sin \left(\theta_{0}-\theta\right)
$$

Only $v_{s d}$ is used as feedback in PLL. Linearising the block about the operating point $\theta_{0}=\theta$ results in a transfer function of gain $V_{m}$. Input to this gain is the difference of $\theta_{0}(s)$ and $\theta(s)$. The resulting model of PLL is shown in Fig. 6.

$$
\theta(s)=T_{p 1}(s) \theta_{0}(s), \quad T_{p 1}=\frac{\frac{V_{m} C_{p}(s)}{s}}{1+\frac{V_{m} C_{p}(s)}{s}}
$$

PLL is supposed to track fundamental component of positive sequence of the input voltage and reject all low-order harmonic content. For this reason, bandwidth of PLL is kept low. But too low a bandwidth is also not desired as $\theta$ will take a long time to respond to sudden changes in $\theta_{0}$.

Initial approximate values of $K_{p}$ and $\omega_{p}$ are given by:

$$
K_{p}^{\prime}=\omega_{p b} / V_{m}, \quad \omega_{p}^{\prime}=\omega_{p b} / 3
$$

$\omega_{p}$ can be kept significantly lower than $\omega_{p}^{\prime}$ since bandwidth is largely determined by $K_{p}$. Frequency response of $\left|T_{p 1}(j \omega)\right|$ and $\angle T_{p 1}(j \omega)$ for $K_{p}=1$ and $\omega_{p}=10 \mathrm{rad} / \mathrm{s}$ are shown in Fig. 7. For the chosen values of the parameters, a bandwidth of about $300 \mathrm{rad} / \mathrm{s}$ is obtained, which is much lower than $\omega_{i b}=$ $3000 \mathrm{rad} / \mathrm{s}$.

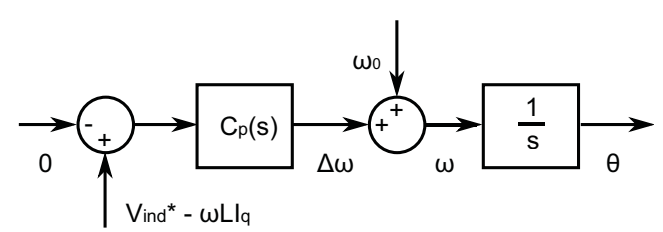

Fig. 8: Block diagram of PLL for proposed sensorless control 


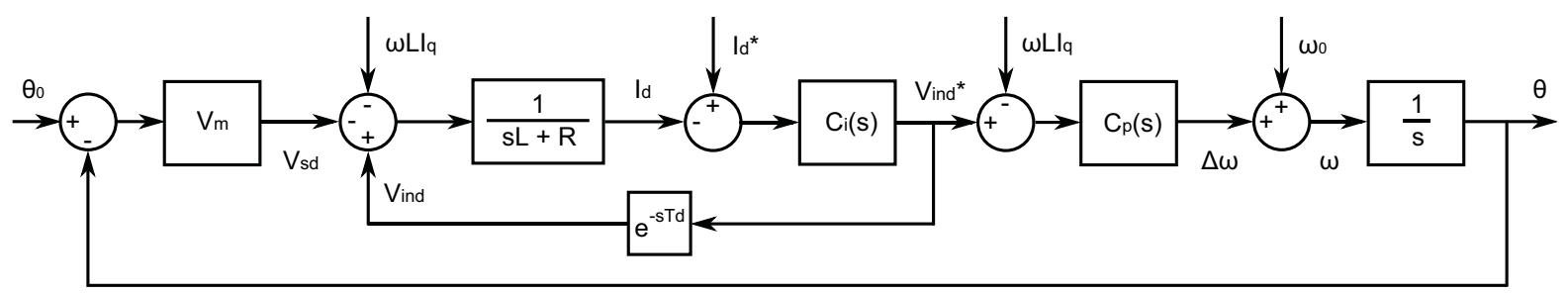

Fig. 9: Linearised model of PLL for proposed sensorless control

\section{Proposed Sensorless Control}

The input voltages are required only by the PLL for obtaining $\theta$. Since PLL response is relatively slow, high frequency components of the input voltages are not required for the operation of PWM rectifier. The limited frequency information required by the PLL can be obtained from the high bandwidth current control loops as shown in this section.

For the PWM rectifier, as shown in Fig. 1, the input voltage equations in $d q$-domain are given by [10]

$$
\begin{aligned}
& v_{s q}=v_{i n q}-R i_{q}+\omega L i_{d}-L \frac{d i_{q}}{d t} \\
& v_{s d}=v_{i n d}-R i_{d}-\omega L i_{q}-L \frac{d i_{d}}{d t}
\end{aligned}
$$

Here, all the voltages and currents are dc quantities in steady state. Hence, last terms in (12) and (13) are zero except during transients. These transients exist for too low a duration for the PLL to respond. Even during transients, magnitude of these terms are insignificant as compared to other terms. Resistive drop is also practically negligible and can be ignored [9]. For the purpose of estimating the input voltage required for the PLL operation, the following equations are proposed to be used:

$$
v_{s q}=v_{i n q}+\omega L i_{d}, \quad v_{s d}=v_{i n d}-\omega L i_{q}
$$

As actual inverter voltage is the reference inverter voltage delayed by a small time (not more than two switching cycles), the q-axis and d-axis voltages can be approximated as follows:

$$
v_{s q} \approx v_{i n q}^{*}+\omega L i_{d}, \quad v_{s d} \approx v_{i n d}^{*}-\omega L i_{q}
$$

The derivative terms in (12) and (13) can be safely neglected as the bandwidth of PLL is around one order lower than that of current control loop. For the purpose of calculating $\theta, v_{s d}$ as obtained from (15) can be directly fed to the PI controller of PLL, as shown in Fig. 8.

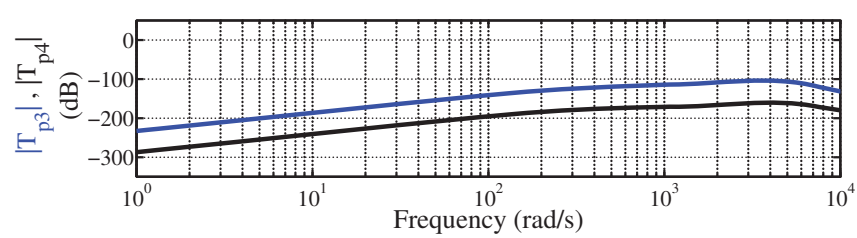

Fig. 10: Bode plot of $T_{p 3}(s)$ and $T_{p 4}(s)$ showing negligible impact of disturbance in proposed PLL.
Complete linearised model of the proposed PLL, as shown in Fig. 9, can be obtained by combining block diagrams from Fig. 2 and Fig. 8. It can be shown that angle $\theta$ in the proposed PLL can be expressed as

$$
\begin{aligned}
\theta(s) & =T_{p 2}(s) \theta_{0}(s)+T_{p 3}(s) I_{d}^{*}(s)+T_{p 4}(s) \omega L I_{q}(s) \\
T_{p 2}(s) & =\frac{\frac{V_{m} C_{i}(s) T_{p 5}(s)}{s L+R}}{1+\frac{V_{m} C_{i}(s) T_{p 5}(s)}{s L+R}}, T_{p 3}(s)=\frac{C_{i}(s) T_{p 5}(s)}{1+\frac{V_{m} C_{i}(s) T_{p 5}(s)}{s L+R}}, \\
T_{p 4}(s) & =\frac{\frac{C_{i}(s) T_{p 5}(s)}{s L+R}-\frac{C_{p}(s)}{s}}{1+\frac{V_{m} C_{i}(s) T_{p 5}(s)}{s L+R}}, T_{p 5}(s)=\frac{\frac{C_{p}(s)}{s}}{1+\frac{e^{-s T_{d} C_{i}(s)}}{s L+R}}
\end{aligned}
$$

Bode plot of $T_{p 2}(s)$ is also shown in Fig. 7 along with that of $T_{p 1}(s)$. The gain and phase plots of $T_{p 2}(s)$ match with those of $T_{p 1}(s)$ for frequencies lower than the bandwidth $\omega_{p b}$. Magnitude plots of $T_{p 3}(s)$ and $T_{p 4}(s)$ are shown in Fig. 10. It can be seen that gains of these are negligible for $I_{d}^{*}(s)$ and $\omega L I_{q}(s)$ to affect the output of PLL. Hence, the performance of the modified PLL is expected to be close to that of conventional sensor based PLL.

\section{Simulation And Experimental Results}

Performance of PWM rectifier controlled using the proposed sensorless vector control method is compared with that of conventional sensor based vector control through simulations and experiments. Hardware and control parameters of the system, as discussed in Section III, for simulations as well as experiments are as given in Table I.

Simulations are conducted using MATLAB-Simulink. For experiments, a general-purpose PWM stack, as mentioned in [14], is used as rectifier. Control of the rectifier is implemented using a Texas Instruments (TI) DSP, TMS320F28377S [15].

\section{A. Simulation Results}

Fig. 11(a) shows the responses of PLL output $\theta$, dc voltage $v_{d c}$, q-axis current $i_{q}$, d-axis current $i_{d}$ and three-phase input current for different transients for sensor based PWM rectifier. Fig. 11(b) shows the corresponding transients responses for the proposed sensorless control. Initially, the rectifier is at no load. Dc bus voltage reference $v_{d c}^{*}$ and d-axis current reference $i_{d}^{*}$ are set at $700 \mathrm{~V}$ and $0 \mathrm{~A}$, respectively. At $t=0.15 \mathrm{~s}$, a step change of $4 \mathrm{~A}$ is given to $i_{d}^{*}$. It is seen that $i_{d}$ responds to the change in reference quickly and settles without any steady state error. During the transients, no changes are observed in $v_{d c}$ and $i_{q}$. At time $t=0.2 \mathrm{~s}, v_{d c}^{*}$ is changed from $700 \mathrm{~V}$ to 800 

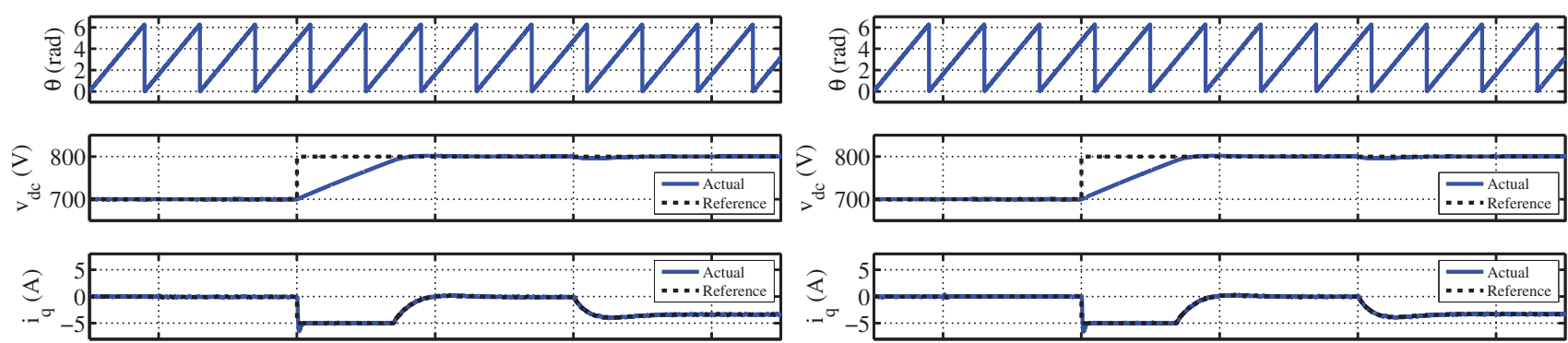

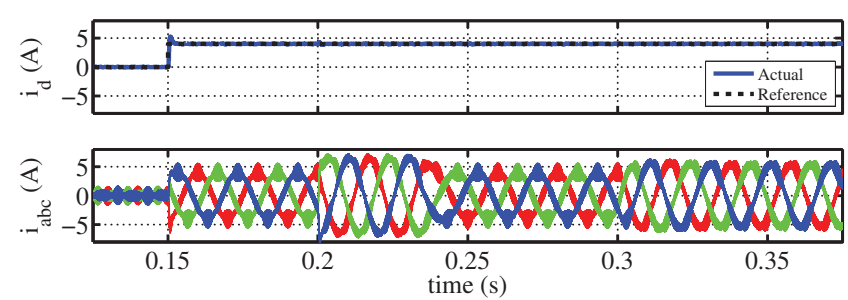

(a)
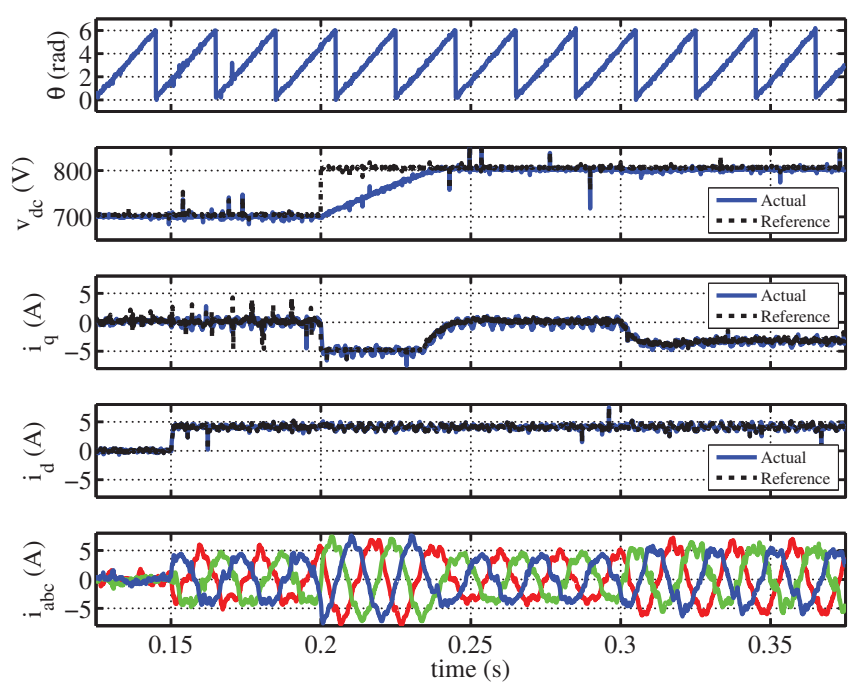

(c)

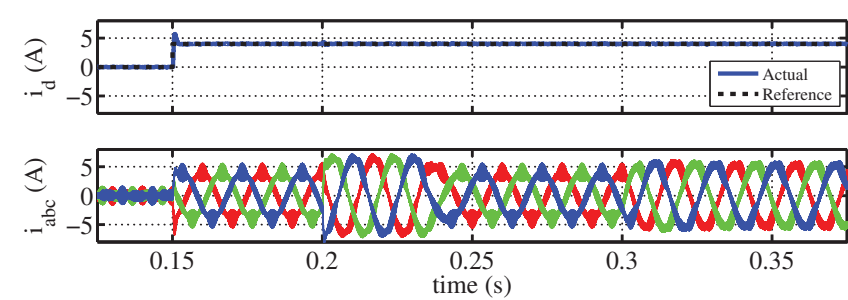

(b)
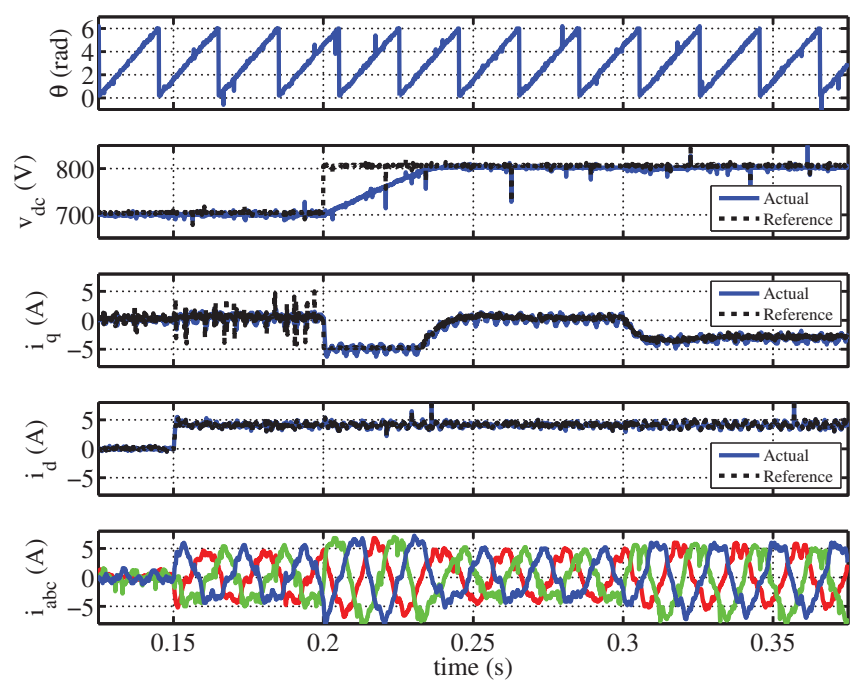

(d)

Fig. 11: Responses of $\theta, v_{d c}, i_{q}, i_{d}$ and input three-phase currents to step changes in $i_{d}^{*}$ at $t=0.15 \mathrm{~s}, v_{d c}^{*}$ at $t=0.2 \mathrm{~s}$ and $\mathrm{dc}$ load at $t=0.3 \mathrm{~s}$ for (a) sensor-based rectifier (simulation) (b) sensorless rectifier (simulation) (c) sensor-based rectifier (experiment) (d) sensorless rectifier (experiment)

V. Voltage controller responds by changing the q-axis current reference $i_{q}^{*}$. Controller saturates to its minimum limit of -5 A. Consequently, q-axis current controller responds by forcing $i_{q}$ to track the reference with zero steady state error; $v_{d c}$ rises linearly until the voltage controller comes out of saturation; $i_{q}^{*}$ again approaches zero as $v_{d c}$ settles at $800 \mathrm{~V}$. During the transition of $v_{d c}$ from $700 \mathrm{~V}$ to $800 \mathrm{~V}$, no transients are observed in $i_{d}$. Finally, a resistive load of $400 \mathrm{ohm}$ is applied to the $\mathrm{dc}$ bus at time $t=0.3 \mathrm{~s}$. Voltage controller responds to the step loading to maintain $v_{d c}$ constant by changing $i_{q}^{*} . i_{q}$ responds to the reference without any steady-state error. For the step loading also, $i_{d}$ is unaffected by the transients in $i_{q}$.

The responses of the sensor-based and sensorless rectifiers are almost identical to one another. Decoupled control is observed in both cases.

\section{B. Experimental Results}

Experimental responses of PLL output $\theta$, dc voltage $v_{d c}$, qaxis current $i_{q}$, d-axis current $i_{d}$ and three-phase input currents corresponding to the simulation responses in Fig. 11(a) are shown in Fig. 11(c). Plots in the figure are as obtained through Digital to Analog Converter (DAC) channels of the DSP. The experimental transient responses obtained are similar to those obtained through simulations.

Fig. 11(d) shows the responses of sensorless rectifier corresponding to those of sensor based rectifier in Fig. 11(c). 

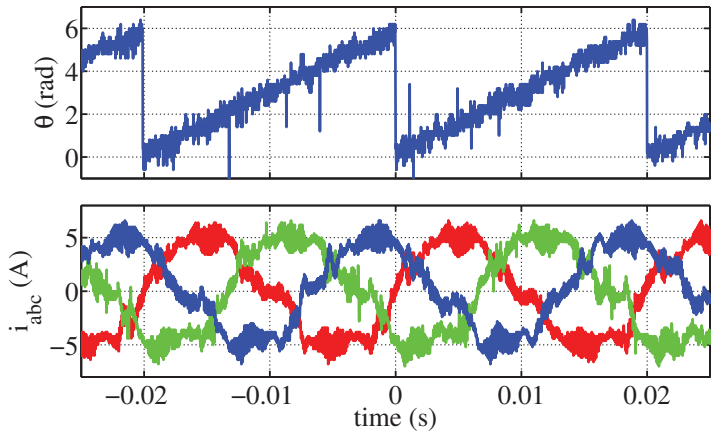

(a)
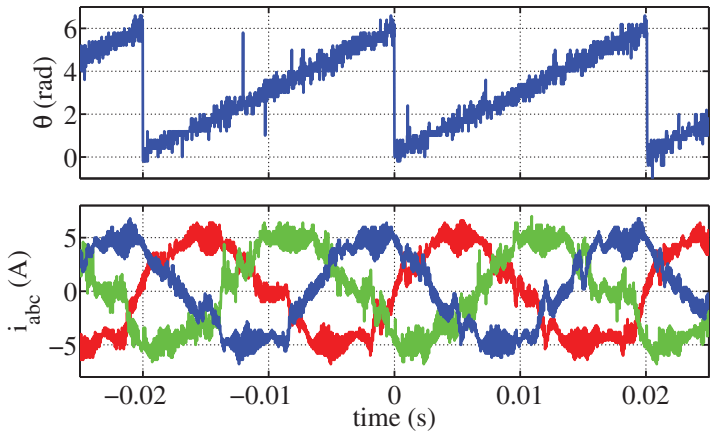

(b)

Fig. 12: Experimental steady state response (PLL angle $\theta$ and three-phase input currents) for $i_{d}^{*}=-5 \mathrm{~A}$ and $v_{d c}^{*}=700 \mathrm{~V}$ at no load of (a) sensor-based (b) sensorless PWM rectifier

Absence of any transients in $i_{q}$ when $i_{d}^{*}$ is changed, and absence of transients in $i_{d}$ when $i_{q}^{*}$ changes as a result of change in $v_{d c}$ or change in dc bus load signify correct estimation of input three-phase voltage. Incorrect estimation would have resulted in losing decoupled control of $i_{d}$ and $i_{q}$.

Steady state three-phase input current waveforms as measured by hall-effect current probes, along with angle $\theta$, for $v_{d c}^{*}=700 \mathrm{~V}$ at no load and $i_{d}^{*}=-5 \mathrm{~A}$ for sensor based rectifier and sensorless rectifier are shown in Fig. 12(a) and Fig. 12(b), respectively. Phase angle shown in Fig. 12(b) is as obtained through sensorless PLL. There is no significant difference between the two plots.

Computation time per switching cycle taken by the DSP for sensor based operation is found to be $24.28 \mu \mathrm{s}$. This time includes Analog to Digital Conversion (ADC), $a b c-d q$ transformation of voltage and current, $d q-a b c$ transformation of inverter reference voltages, and execution times of PLL, voltage control loop and current control loop. The corresponding time taken for sensorless operation is $22.31 \mu \mathrm{s}$. Computation time for sensorless operation is lower than that for sensor based operation on account of reduction in number of analog to digital conversions and elimination of $a b c-d q$ transformation of input voltage.

\section{CONCLUSION}

A simplified input voltage sensorless control for PWM rectifier is proposed in this paper. The proposed method achieves sensorless operation by making simple modification to the conventional sensor-based vector control. Instead of sensing the input voltages for obtaining the synchronously rotating phase angle, input voltages are estimated directly in $d q$-domain only through algebraic equations. Unlike the existing sensorless control methods, the proposed method does not require extensive mathematical computations. It retains the performance of the sensor-based control without any additional controller design. Computation time required by the proposed control is even lower than that for the sensor-based control.

\section{REFERENCES}

[1] A. Marzouki, M. Hamouda and F. Fnaiech, "A review of PWM voltage source converters based industrial applications," in Proc. Int. Conf. on
Electrical Systems for Aircraft, Railway, Ship Propulsion and Road Vehicles (ESARS), Aachen, 2015, pp. 1-6.

[2] M. B. Ketzer and C. B. Jacobina, "Sensorless Control Technique for PWM Rectifiers With Voltage Disturbance Rejection and Adaptive Power Factor," IEEE Trans. Ind. Electron., vol. 62, no. 2, pp. 1140-1151, Feb. 2015.

[3] H. Yoo, J. H. Kim and S. K. Sul, "Sensorless Operation of a PWM Rectifier for a Distributed Generation," IEEE Trans. Power Electron., vol. 22, no. 3, pp. 1014-1018, May 2007.

[4] S. Hansen, M. Malinowski, F. Blaabjerg and M. P. Kazmierkowski, "Sensorless control strategies for PWM rectifier," in Proc. IEEE Applied Power Electronics Conference and Exposition, New Orleans, LA, USA, 2000, pp. 832-838 vol.2.

[5] M. Malinowski and S. Bernet, "A Simple Voltage Sensorless Active Damping Scheme for Three-Phase PWM Converters With an $L C L$ Filter," IEEE Trans. Ind. Electron., vol. 55, no. 4, pp. 1876-1880, April 2008.

[6] H. Zhang, X. Zhu, J. Shi, L. Tan, C. Zhang and K. Hu, "Study on PWM Rectifier without Network Voltage Sensor Based on Virtual Flux Delay Compensation Algorithm," IEEE Trans. Power Electron, Early Access.

[7] J. G. Norniella et al., "Improving the Dynamics of Virtual-Flux-Based Control of Three-Phase Active Rectifiers," IEEE Trans. Ind. Electron., vol. 61, no. 1, pp. 177-187, Jan. 2014.

[8] Y. Tao, Q. Wu, L. Wang and W. Tang, "Voltage sensorless predictive direct power control of three-phase PWM converters," IET Power Electron., vol. 9, no. 5, pp. 1009-1018, 4202016.

[9] Y. Cho and K. Lee, "Virtual-Flux-Based Predictive Direct Power Control of Three-Phase PWM Rectifiers With Fast Dynamic Response," IEEE Trans. Power Electron., vol. 31, no. 4, pp. 3348-3359, April 2016.

[10] A. Rahoui, A. Bechouche, H. Seddiki and D. O. Abdeslam, "Grid Voltages Estimation for Three-Phase PWM Rectifiers Control Without AC Voltage Sensors," IEEE Trans. Power Electron., vol. 33, no. 1, pp. 859-875, Jan. 2018.

[11] D. E. Quevedo, R. P. Aguilera, M. A. Perez, P. Cortes and R. Lizana, "Model Predictive Control of an AFE Rectifier With Dynamic References," IEEE Trans. Power Electron., vol. 27, no. 7, pp. 3128-3136, July 2012.

[12] A. M. Bozorgi, H. Gholami-Khesht, M. Farasat, S. Mehraeen and M. Monfared, "Voltage sensorless improved model predictive direct power control for three-phase grid-connected converters," in Proc. IEEE Energy Conversion Congress and Exposition (ECCE), Cincinnati, OH, 2017, pp. 4957-4963.

[13] J. Siva Prasad, T. Bhavsar, R. Ghosh, and G. Narayanan, "Vector control of three-phase ac/dc front-end converter," Sadhana, vol. 33, no. 5, pp. 591-613, Oct. 2008.

[14] D. Venkatramanan, A. K. Adapa and V. John, "Design and Comparative Study of Discrete and Module based IGBT Power Converters," in Proc. 7th National Power Electron. Conf. (NPEC), Mumbai, India, 2015.

[15] Texas Instruments, "TMS320F2837xS Delfino Microcontrollers," TMS320F28377S datasheet, Aug. 2014 [Revised May 2018]. 